\title{
TRANSMISSION OF THE L-ZAGREB MUMPS VACCINE VIRUS, CROATIA, $2005-2008$
}

\author{
B Kaic (bernard.kaic@hzjz.hr)1, I Gjenero-Margan¹, B Aleraj¹, S Ljubin-Sternak², T Vilibic-Cavlek², S Kilvain³ , I Pavic ${ }^{4}$, \\ D Stojanovic ${ }^{5}$ A Ilic ${ }^{6}$ \\ 1. Croatian Institute of Public Health, Department of Infectious Disease Epidemiology, Zagreb, Croatia \\ 2. Croatian Institute of Public Health, Virology Department, Croatian Institute of Public Health, Zagreb, Croatia \\ 3. Primary health care paediatrician, Rijeka, Croatia \\ 4. Department of Infectious Diseases, University Hospital, Rijeka, Croatia \\ 5. Department of Epidemiology, Primorje-Gorski Kotar County Institute of Public Health, Rijeka, Croatia \\ 6. Department of Epidemiology, Vukovar-Srijem County Institute of Public Health, Vinkovci, Croatia
}

\begin{abstract}
We report on three cases of symptomatic transmission of the $\mathrm{L}$-Zagreb mumps vaccine virus from three vaccinated children to five adult contacts. The five contact cases were parents of the vaccinated children and presented with parotitis and in one case also with aseptic meningitis. The etiology of the contacts' illness was determined by viral culture, genomic sequencing, serology and epidemiological linking. Two of the vaccinated children developed vaccine associated parotitis as an adverse event three weeks following immunization. Symptoms in contact cases developed five to seven weeks after the vaccination of the children. The five contact cases, as well as the three children with adverse events recovered completely. The children had been vaccinated with MMR vaccine produced by the Institute of Immunology Zagreb, each of them with a different lot. One of the possible explanations for these adverse events is that the very low levels of wild mumps virus circulation in the last decade, combined with waning immunity in those who received one dose of vaccine or suffered from mumps in childhood, resulted in susceptible young adults and that this unique epidemiological situation allows us to detect horizontal transmission of mumps vaccine virus.
\end{abstract}

\section{Introduction}

Vaccination against mumps was introduced into the Croatian vaccination schedule in 1976 for all children at the age of 12 months [1]. In 1994, a second dose of mumps vaccine was added to the vaccination schedule for seven-year-old children [1]. Mumps vaccine is delivered as a trivalent measles - mumps - rubella (MMR) vaccine. The mumps component of the vaccine is prepared from the $L$-Zagreb vaccine strain. Since the introduction of the vaccine, vaccination coverage has constantly been higher than $90 \%$, ranging from 93 to $98 \%$, both for primary vaccination before the second birthday and for revaccination before the eighth birthday.

Due to high vaccination coverage, the incidence of mumps declined from over 10,000 cases to less than 100 cases annually (Figure). In 2007, only 77 cases of mumps were reported (incidence of 1.7 per 100,000). Mumps cases are subject to mandatory reporting on the basis of clinical suspicion, regardless of the laboratory confirmation.
Reporting of adverse events following vaccination is mandatory, as is the vaccination itself. Since 1994, we have recorded annually 50 to 70 cases of vaccine-associated parotitis and five to 15 cases of vaccine-related aseptic meningitis in vaccine recipients. Over 80,000 doses of MMR vaccine are administered annually.

\section{Deascription of cases}

The first case was reported in October 2005 from Rijeka. A healthy 14-month-old boy was routinely vaccinated on in mid-September with MMR. Twenty-six days later, he developed unilateral, febrile parotitis. Routine laboratory investigation revealed elevated serum amylases. In late October, six weeks after the child had received the vaccine, his mother was hospitalised due to fever, unilateral parotitis and headache. Lumbar puncture revealed pleocytosis of the cerebrospinal fluid (CSF) and viral meningitis was suspected. By viral culture performed at the Croatian Institute Public Health, mumps virus was isolated on Vero cells from the CSF and confirmed by indirect immunofluorescent assay (IFA) (Light Diagnostics, Temecula, CA). The isolate was subsequently characterised by genomic sequencing and comparing the genome with the reference

\section{F I G U R E}

Incidence of mumps in Croatia 1976-2006

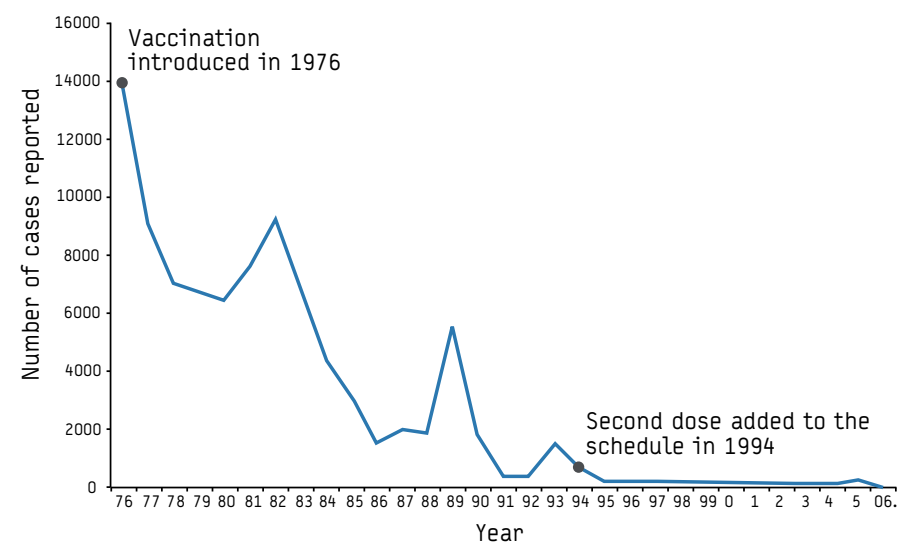


sequences (GenBank NIH, Bethesda) performed at the Institute of Immunology Zagreb, as L-Zagreb vaccine strain.

The mother was born in the mid-1970s. According to her medical records, she was not vaccinated against mumps and does not have a history of parotitis. There are no other family members living in the same household. Both mother and child recovered completely. During the last trimester of 2005, there have been no reports of mumps from the Rijeka region.

The second case was reported in October 2007 in Zagreb. A healthy 17 -month-old girl was routinely vaccinated in late August. Three weeks later, she had an episode of fever and cough, which was not considered related to vaccination. She had a white blood cell count performed at that time, which revealed leukopenia with relative lymphocytosis. Six weeks after vaccination, the child's mother developed bilateral febrile parotitis, and four days after that the father also developed bilateral parotitis. Sera of the parents, collected three days later, were tested by ELISA for cytomegalovirus (CMV) antibodies and IFA for mumps virus antibodies (Viro-immun) and Epstein-Barr virus (EBV) antibodies. Both parents were IgG positive and IgM negative for CMV and EBV antibodies. The father was IgM and IgG positive, while the mother was low IgM positive and IgG positive for mumps virus antibodies.

The isolation of mumps virus on Vero cells from urine specimens and salivary duct swabs of both parents was attempted at the Croatian Institute Public Health. Mumps virus was only isolated from the mother's salivary gland duct swab, and confirmed by IFA (Light Diagnostics, Temecula, CA). The isolate was subsequently characterised, by genomic sequencing and comparing the genome with the reference sequences (GenBank NIH, Bethesda) performed at the Institute of Immunology Zagreb, as L-Zagreb vaccine strain.

The mother, born in 1970, received all routine childhood vaccinations according to her mother's statement, but no evidence of vaccination was found in medical records. The father, also born in the mid-1970s, had mumps as a child according to his mother's statement, but no medical documentation was found to support that either. There were no other family members living in the same household. Both parents recovered completely. During September and October 2007, mumps activity was very low in Zagreb and its surroundings, with only two cases reported, both geographically distant from the residence of this family.

The third case was reported in January 2008 in Zupanja. A healthy 15 -month-old boy was routinely vaccinated in midDecember 2007. Sixteen days later, he developed unilateral, febrile parotitis. No laboratory testing was performed. On 15 January, the mother developed bilateral, painless, afebrile parotitis and three days later, the father developed unilateral afebrile parotitis. Sera of both parents was collected four days after that, and tested by IFA for mumps virus (Vero-immun) antibodies. Both parents were IgG positive and IgM negative for mumps virus antibodies.

The isolation of the mumps virus on Vero cells from urine specimens and salivary duct swabs of the father was performed at the Croatian Institute of Public Health. The mumps virus was isolated from his salivary gland duct swab and confirmed by IFA (Light Diagnostics, Temecula, CA). The isolate was subsequently characterised by genomic sequencing and comparing the genome with the reference sequences (GenBank NIH, Bethesda) performed at the Institute of Immunology Zagreb, as L-Zagreb vaccine strain.

The RT-PCR for mumps virus performed on the father's urine specimen was negative, while the RT-PCR testing of the father's salivary gland duct swab was positive for mumps virus RNA.

There is no information on the vaccination and medical history of the parents.

A three-year old brother of the vaccinated child lives in the same household. He was vaccinated with MMR at the age of one year and was healthy throughout the period between December 2007 and February 2008

During January and February, there have not been any reports of mumps from Zupanja region. Genetic characterization of the three isolates described above was performed by the sequence analysis of the most variable gene of the mumps virus, small hydrophobic gene, and comparing the nucleotide sequence with the reference sequences (GenBank NIH, Bethesda) as described previously $[2,3]$.

Discussion

We demonstrated horizontal transmission of the L-Zagreb mumps vaccine virus, which resulted in symptomatic illness in contacts.

For three of the five parents who developed parotitis, there is direct evidence of the vaccine strain recovered from the contact cases, confirmed by genomic sequencing of the isolated virus. In the two symptomatic parents without isolation of the mumps virus, the incubation period and the fact that there are no mumps cases in the region are in favour of a causal relationship between the child's vaccination and the parents' parotitis.

Although it is well known that some live attenuated vaccine strains can be transmitted to contacts, e.g. oral polio, varicellazoster, there are only few reports of transmission of mumps vaccine viruses to contacts $[4,5,6]$. Searching the literature, we found only two published papers describing horizontal transmission of a mumps vaccine virus, apart from our own report two years ago [6] Sawada and colleagues demonstrated the asymptomatic horizontal transmission of the Urabe strain [4], while Atrasheuskaya and colleagues demonstrated the symptomatic transmission of the Leningrad-3 mumps vaccine strain [5].

We are exploring possible explanations for the three events reported in this communication. The three cases we described do not represent a cluster, since they occurred in different geographical areas, there is no clustering in time and three separate vaccine lots are involved. Therefore, a mistake in the production of a vaccine lot can be ruled out as an explanation for these events. However, we can not rule out a de-attenuation of the vaccine virus that has been propagated to several lots. The unchanged incidence of other adverse events caused by this vaccine virus (vaccine associated parotitis and aseptic meningitis) does not suggest a general deattenuation.

It would be useful to know if the rates at which the parents contract disease after having contact with their vaccinated children are increasing, since this would point towards a de-attenuation of the vaccine. 
We do not currently have a sufficient number of reports to determine if the described cases represent a rise in incidence. Careful surveillance of adverse events following immunisation will soon provide an answer to this question. Following acknowledgement of the described cases, a letter was sent to all vaccine providers in the country informing them of the possibility of transmission of the vaccine virus to contacts, resulting in illness.

We believe that these events are a consequence of a change in population susceptibility rather than in the properties of the vaccine virus. Our hypothesis is that a horizontal transmission of the mumps vaccine virus has always been occurring at very low rates, but we were not able to detect it.

Owing to the fact that we have a very favourable epidemiological situation with very low levels of wild mumps virus circulation in the population in the last decade, adolescents and young adults who were vaccinated only once are susceptible to mumps because of waning immunity and the lack of natural boosters that natural infection provides. This gives rise to the accumulation of susceptible young adults. Schmid et al. recently reported on a mumps outbreak in Austria and were able to show that 68 of their patients were vaccinated only once [7].

The occurrence of adverse effects requires a critical re-evaluation of the appropriateness of the use of the L-Zagreb strain as a vaccine strain for MMR vaccination in countries with a low level of wild mumps virus transmission. The risk of vaccine side-effects is the leading argument of groups opposing MMR vaccination. It is therefore important to inform the public about the relative safety of the vaccine and of possible complications of mumps, namely meningitis, orchitis and pancreatitis.

In countries with higher levels of wild mumps virus transmission and occasional outbreaks young adults' immunity is boosted through contact with the wild virus. Therefore, in such settings waning immunity following one dose of mumps vaccine can not be demonstrated and, thanks to natural booster, it does not lead to accumulation of susceptible young adults.

\section{Acknowledgements}

The authors are grateful to the Institute of Immunology Zagreb for performing the genomic sequencing of the mumps virus isolates.

The authors are grateful to Professor J Pavlic for technical help in preparing the manuscript.

\section{References}

1. Borcic B, Mazuran R, Kaic B. Immunity to measles in the Croatian population. Eur J Epidemiol. 2003;18(11):1079-83.

2. Santak M, Kosutic-Gulija T, Tesovic G, Ljubin-Sternak S, Gjenero-Margan I, Betica-Radic L, et al. Mumps virus strains isolated in Croatia in 1998 and 2005: Genotyping and putative antigenic relatedness to vaccine strains. J Med Virol. 2006;78(5):638-43.

3. Jin L, Rima B, Brown D, Orvell C, Tecle T, Afzal M, et al. Proposal for genetic characterisation of wild-type mumps strains: preliminary standardisation of the nomenclature. Arch Virol. 2005;150(9):1903-9.

4. Sawada H, Yano S, Oka Y, Togashi T. Transmission of Urabe mumps vaccine between siblings. Lancet. 1993;342(8867):371

5. Atrasheuskaya AV, Neverov AA, Rubin S, Ignatyev GM. Horizontal transmission of the Leningrad-3 live attenuated mumps vaccine virus. Vaccine. 2006;24(10):1530-6. Epub 2005 0ct 18.
6. Kaic B. Ed. Adverse Events Following vaccination in Croatia in 2005 [in Croatian]. Zagreb: Croatian Institute of Public Health, 2006; 5. Available from: http://www.hzjz.hr/epidemiologija/nuspojave2005.pdf

7. Schmid D, Holzmann H, Alfery C, Wallenko H, Popow-Kraupp Th, Allerberger F. Mumps outbreak in young adults following a festival in Austria, 2006. Euro Surveill 2008;13(7). Available from: http://www.eurosurveillance.org/edition/ v13n07/080214_6.asp

This article was published on 17 April 2008.

Citation style for this article: Kaic B, Gjenero-Margan I, Aleraj B, Ljubin-Sternak S, Vilibic-Cavlek T, Kilvain S, Pavic I, Stojanovic D, Ilic A. Transmission of the L-Zagreb mumps vaccine virus, Croatia, 2005-2008. Euro Surveill. 2008;13(16):pii=18843. Available online: http://www.eurosurveillance.org/ViewArticle.aspx?ArticleId=18843 